11

\title{
Формирование и оптические свойства нанорешеток на поверхности фторида кальция, генерируемых при фемтосекундном лазерном воздействии
}

\author{
(C) А.Е. Рупасов ${ }^{1,2}$, П.А. Данилов ${ }^{1,2}$, А.А. Ионин ${ }^{1}$, Н.А. Смирнов ${ }^{1,2}$, С.И. Кудряшов ${ }^{1,2}$, А.A. Руденко ${ }^{1}$, \\ А.Н. Путилин ${ }^{1}$, Р.А. Заколдаев ${ }^{2}$ \\ ${ }^{1}$ Физический институт им. П.Н. Лебедева, \\ 119991 Москва, Россия \\ ${ }^{2}$ Университет ИТМО, \\ 197101 Санкт-Петербург, Россия \\ e-mail: jkljnb16@gmail.com
}

Поступила в редакцию 28.03.2021 г.

В окончательной редакции 28.03.2021 г.

Принята к публикации 27.04.2021 г.

Фемтосекундное лазерное структурирование диэлектриков является актуальной задачей для создания оптических элементов. В данной работе выполнялось фемтосекундное лазерное наноструктурирование поверхности фторида кальция с образованием самоорганизующихся периодических решеток, которые обладают субволновым периодом порядка 200 и $350 \mathrm{~nm}$. В процессе работы была установлена зависимость периода структур от длины волны лазерного излучения, подтвержденная результатами моделирования. Структуры показывают уменьшение пропускания во всем видимом диапазоне преимущественно из-за дифракции и рассеяния света.

Ключевые слова: прямая лазерная запись, фемтосекундные лазерные импульсы, поверхностные функциональные нано- и микрооптические структуры, дифракция.

DOI: $10.21883 /$ OS.2021.08.51204.1998-21

\section{Введение}

В последние годы активно развивается область прямой лазерной записи оптических и других функциональных элементов в объеме и на поверхности широкозонных диэлектриков. При взаимодействии лазерного импульса с материалом происходит процесс нелинейного поглощения. Для того чтобы реализовать процесс нелинейного поглощения, напряженность электрического поля в лазерном импульсе должна быть сопоставима с полем, которое связывает электроны в атомах [1-3]. Чтобы достичь такой напряженности поля, требуется высокая интенсивность лазерного излучения. Под воздействием фемтосекундных лазерных импульсов в зависимости от параметров излучения можно получить различные модификации в диэлектриках. К таким структурам относятся области уплотнения и разуплотнения материала, создание точечных дефектов и т.д. [4,5]. Лазерная обработка диэлектрика находит свое применение в таких приложениях как запись волноводов [6], изготовление двулучепреломляющих элементов [7], трехмерная оптическая память [8], а также в новых областях как голографические запись [9] и 5D оптическая память [10]. Различия заключаются в плотности вкладываемой лазерной энергии, которая была оценена в работе [11], для получения периодических нанорешеток [12], формирования полых нанопор [13] или сверления микроканалов [14] с различным количеством удаления абляционного материала.
Фторид кальция - широкозонный диэлектрик, который обладает достаточно низким показателем преломления $(n \approx 1.4)$, при этом имеет достаточно высокую оптическую прозрачность в широком спектральном диапазоне 0.15-9 $\mu \mathrm{m}$. Это делает его более перспективным материалом по сравнению со стеклом (пропускание от $0.2-1.2 \mu \mathrm{m})$ для создания на его основе оптических элементов путем прямой фемтосекундной лазерной записи.

В данной работе рассматривается формирование в флюорите самоорганизующихся периодических наноструктур, период которых напрямую связан с длиной волны лазерного излучения. Нанометровые решетки имеют потенциальное применение в плазмонике, фотонике, оптоэлектронике и биооптических устройствах. Тип структуры, в том числе зависит от поляризации лазерного излучения. Для получения вытянутых периодических структур поляризация лазерного излучения должна быть сонаправлена с направлением сканирования. Различают несколько типов получаемых модификаций. В работе [15] на поверхности фторида кальция были получены структуры с периодом порядка длины волны - $625 \mathrm{~nm}$, а в нашей работе мы хотим рассказать о лазерном формировании субволновых решеток, период которых может быть порядка 200 или $350 \mathrm{~nm}$. Механизмы образования поверхностных структур в металлах, диэлектриках, полупроводниках основаны на различных 


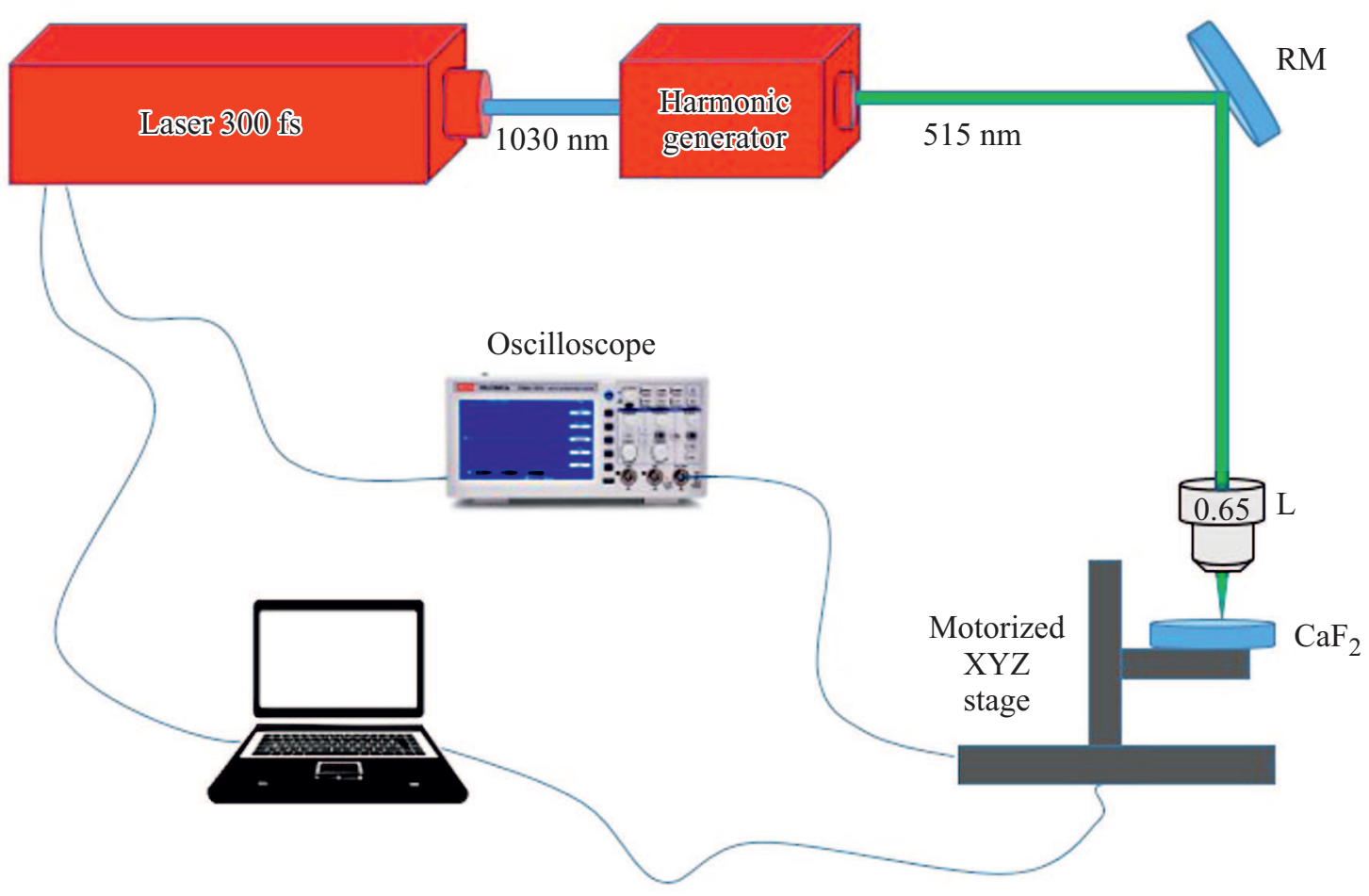

Рис. 1. Схематическая иллюстрация экспериментальной установки: Laser — лазер Satsuma; Harmonic generator — oптический генератор второй гармоники $(515 \mathrm{~nm}) ; \mathrm{RM}-$ отражающее зеркало; L - микрообъектив с числовой апертурой NA $=0.65$; Motorized XYZ stage - трехосевая моторизованная платформа Standa; Oscilloscope - осциллограф, для синхронизации моторизированной подвижки и лазера.

теориях, однако не существует достаточного понимания механизма их образования.

Ранее в кратком сообщении [16] мы представили экспериментальные данные о возможности формирования решеток под действием лазерного импульса. Настоящую работу дополняем данными об оптических свойствах материала и предполагаемыми возможными механизмами образования решеток, с применением численного моделирования, которые хорошо согласуются с результатами эксперимента.

\section{Экспериментальная часть}

Источником фемтосекундных лазерных импульсов была лазерная система Satsuma [17], основной является иттербиевый волоконный лазер. Установка включает в себя генератор, многопроходный усилитель, выходной компрессор и акустооптический модулятор. В лазере используется диод накачки с длиной волны $850 \mathrm{~nm}$. Управление осуществляется через контрольную панель или программу управления на внешнем компьютере. Экспериментальная установка позволяет получать импульсы длительностью $300 \mathrm{fs}$, частотой до $500 \mathrm{kHz}$. На выходе системы энергия в импульсе составляет до $10 \mu \mathrm{J}$ для длины волны $1030 \mathrm{~nm}$, а для $515 \mathrm{~nm}$ до $3.5 \mu \mathrm{J}$.

В рамках данной работы структуры были записаны на поверхности фторида кальция излучением с длиной волны 515 и $1030 \mathrm{~nm}$ с помощью фемтосекундной лазерной установки Satsuma. Лазерное излучение фокусировалось с помощью объектива микроскопа с числовой апертурой 0.65 (рис. 1) на поверхность в пятно радиусом $R_{1 / e}=1.8 \pm 0.2 \mu \mathrm{m}$ для длины волны $515 \mathrm{~nm}$ и гауссовым пространственным распределением по энергии. Запись выполнялась при нормальном падении излучения и различных ориентациях линейной поляризации лазерного излучения относительно направления сканирования. Изменение поляризации импульсов выполнялось с помощью поворота полуволновых пластинок.

Образец представляет собой пластинку (срез монокристалла, с кубической решеткой 111) фторида кальция толщиной $2 \mathrm{~mm}$ и диаметром $15 \mathrm{~mm}$. Пластинка крепилась на держателе с регулировкой угла наклона, держатель был установлен на моторизированной трехосевой платформе Standa. Структуры были записаны в области $100 \times 100 \mu \mathrm{m}$. При записи периодических микроструктур оптимальная энергия в импульсе составляла $64 \mathrm{~nJ}$. Для достижения лучшей однородности структуры число импульсов в точку должно превышать 10000 . В таких режимах обработки были использованы скорости записи от 20 до $100 \mu \mathrm{m} / \mathrm{s}$.

Для получения наномасштабного изображения поверхности использовался автоэмиссионный растровый электронный микроскоп JSM-7001F (JEOL). В высоковакуумном микроскопе используется катод Шотки, 

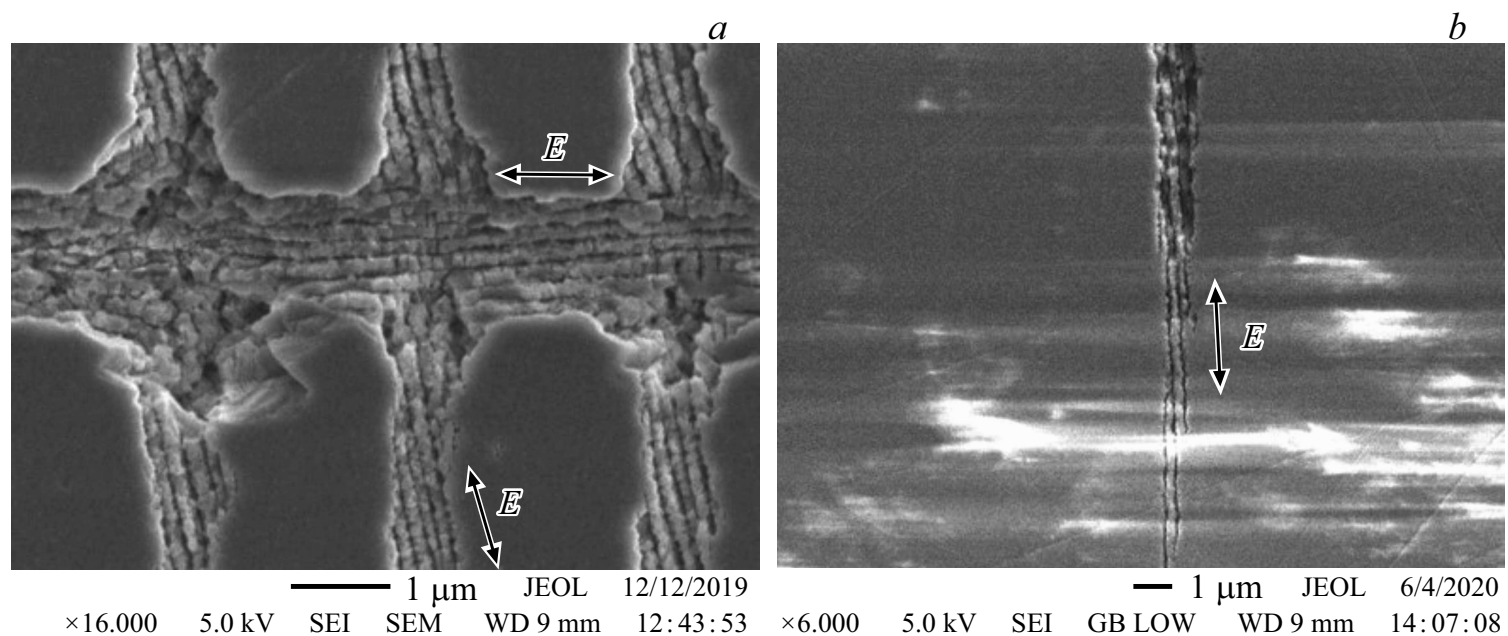

Рис. 2. Снимки СЭМ поверхностных структур на $\mathrm{CaF}_{2}$, записанных при обработке на длине волны $515(a)$ и $1030 \mathrm{~nm}(b)$.

позволяя добиться разрешения порядка $2 \mathrm{~nm}$. При исследовании диэлектрических образцов возникают сложности с зарядом поверхности. Для решения данной проблемы применялись следующие методы: запыление поверхности образца тонкой пленкой металла; использование EBSD-детектора; применение низких ускоряющих напряжений $<5 \mathrm{keV}$ и токов меньше $30 \mathrm{pA}$.

С помощью микроскопа-спектрофотометра ЛОМО МСФУ-К с объективом с числовой апертурой $\mathrm{NA}=0.65$ и диафрагмой $0.1 \mathrm{~mm}$ были измерены спектры отражения и пропускания модифицированных участков в видимом спектральном диапазоне. В качестве источника света использовалась галогеновая лампа.

\section{Экспериментальные результаты и их обсуждение}

\section{1. Топография наноструктур}

Методами сканирующей электронной микроскопии были получены снимки периодических поверхностных самоорганизующихся структур, записанных на поверхности фторида кальция с помощью фемтосекундного лазера для двух длин волн 515 и $1030 \mathrm{~nm}$ (рис. 2).

При длине волны лазерного излучения в $515 \mathrm{~nm}$ период структур составляет в пределе от 150 до $250 \mathrm{~nm}$ (рис. 2, a), а при длине волны в $1030 \mathrm{~nm}$ период структур составляет от 300 до $400 \mathrm{~nm}$ (рис. 2, $b$ ). Могут быть получены скрещенные решетки (рис. 2, a), при повороте поляризации на $90^{\circ}$ период решетки сохраняется. В случае использования радиальной и азимутальной поляризации период структур может отличаться, как было показано в работе [18].

В общем случае образование поверхностных периодических структур может быть связано с интерференцией как между падающей электромагнитной волной

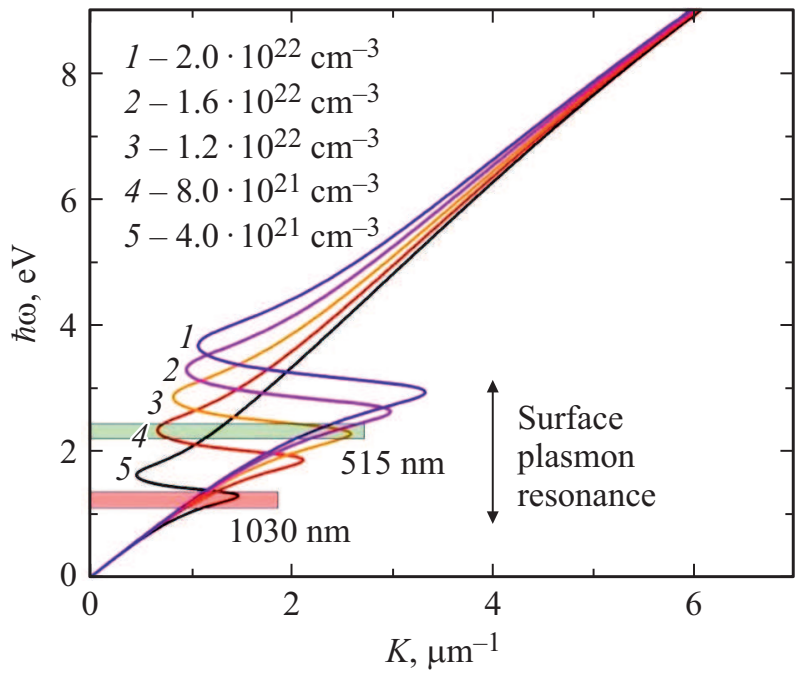

Рис. 3. Дисперсионные кривые для поверхностных электромагнитных волн на фотовозбужденной поверхности фторида кальция при различных плотностях электрон-дырочной плазмы.

(источником света) и поверхностной рассеянной электромагнитной (плазмон-поляритонной) волной с волновым числом $K \geq 1 / \lambda_{\text {las }}$ (решетки с околоволновыми периодами) [19], так и при нормальном падении лазерного излучения, между коротковолновыми поверхностными плазмонами с противонаправленными волновыми векторами и одинаковыми высокими волновыми числами $K \gg 1 / \lambda_{\text {las }}$ (глубоко-субволновые решетки) [20]. Пороговым условием возбуждения поверхностных плазмонов и плазмон-поляритонов является равенство $\operatorname{Re}\left[\varepsilon_{\mathrm{CAF} 2}^{*}\right]=-\operatorname{Re}\left[\varepsilon_{\mathrm{AIR}}\right][21]$, где $\operatorname{Re}\left[\varepsilon_{\mathrm{CAF} 2}^{*}\right]-$ действительной часть диэлектрической проницаемости возбужденного флюорита [22] и $\varepsilon_{\text {AIR }} \approx 1$, а соответствующая критическая плотность электрон-дырочной плазмы, обеспечивающая описываемую моделью Друде 


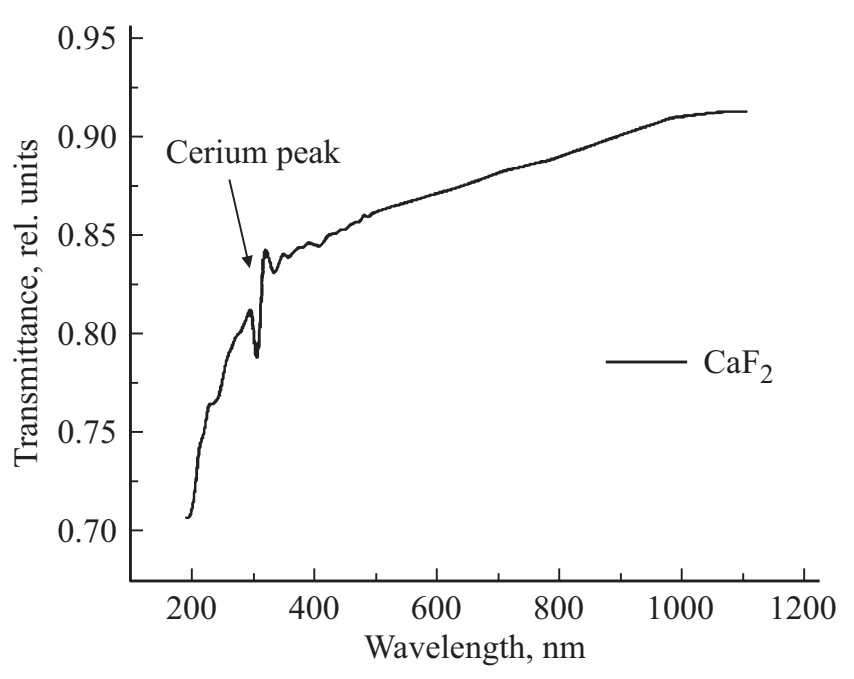

Рис. 4. Спектр пропускания пластинки фторида кальция.

металлизацию материала, составляет $\approx 8 \cdot 10^{21} \mathrm{~cm}^{-3}$ для $515 \mathrm{~nm} \mathrm{и} \approx 2 \cdot 10^{21} \mathrm{~cm}^{-3}$ для $1030 \mathrm{~nm} \mathrm{[11]).} \mathrm{С} \mathrm{учетом}$ данных параметров, а также массы электрона в зоне проводимости, равной массе свободного электрона, и частоте рассеяния носителей порядка плазменной частоты $\left(\sim 10 / \omega_{\mathrm{pl}}\right)$ [23], по аналогии с работой [24] были рассчитаны дисперсионные соотношения для поверхностных электромагнитных волн на фотовозбужденной поверхности фторида кальция при различных плотностях электрон-дырочной плазмы (рис. 3). Данная модель справедлива для двух однородных полубесконечных сред. Если рассматривать фотовозбужденный слой диэлектрика конечной толщины, то условия возбуждения меняются, что потребует правок в законы дисперсии [25].

Расчеты показали, что с ростом плотности электрондырочной плазмы поверхностный плазмонный резо- нанс двигается вверх по энергии кванта и для длины волны $1030 \mathrm{~nm}$ достигается при плотности плазмы менее $4 \cdot 10^{21} \mathrm{~cm}^{-3}$, а волновое число существенно демпфированных плазмонов [20] составляет при этом $K \leq 1.5 \mu \mathrm{m}^{-1}$ (рис. 3). В результате интерференции поверхностных плазмонов это дает период интерференционной стоячей волны поля на поверхности $1 / 2 K \geq 350 \mathrm{~nm}$, что хорошо согласуется с экспериментально наблюдаемым периодом нанорешеток от 300 до $400 \mathrm{~nm}$. Аналогично для длины волны $515 \mathrm{~nm}$ поверхностный плазмонный резонанс достигается при плотности плазмы выше $8 \cdot 10^{21} \mathrm{~cm}^{-3}$, а волновое число существенно демпфированных плазмонов составляет при этом $K \approx 2.5 \mu \mathrm{m}^{-1}$ (рис. 3). Соответственно период интерференционной стоячей волны достигает $1 / 2 K \approx 200 \mathrm{~nm}$, что также хорошо согласуется с экспериментальными данными (от 150 до $250 \mathrm{~nm}$ ). Результаты моделирования показывают, что при использовании третьей $(343 \mathrm{~nm}, 3.5 \mathrm{eV})$ и четвертой $(257 \mathrm{~nm}$, $4.6 \mathrm{eV})$ гармоник фемтосекундного лазерного излучения для широкозонного флюорита с пренебрежимым межзонным поглощением на этих длинах волн в рамках того же механизма интерференции поверхностных плазмонов можно получить нанорешетки с периодами $\sim 100 \mathrm{~nm}$.

\section{2. Спектроскопия}

Спектр пропускания исходного образца (рис. 4) был измерен с помощью спектрофотометра СФ-2000. Исходя из полученных данных можно сделать выводы, что в образце присутствуют примеси редкоземельных ионов.

В спектре пропускания наблюдается полоса поглощения на $302 \mathrm{~nm}$ [26], пики в этой области являются характерными для ионов $\mathrm{Ce}^{2+}$ и $\mathrm{Ce}^{3+}$. Церий является типичной примесью флюорита. Вероятно, в процессе роста кристалла шихта была недостаточно очищена,
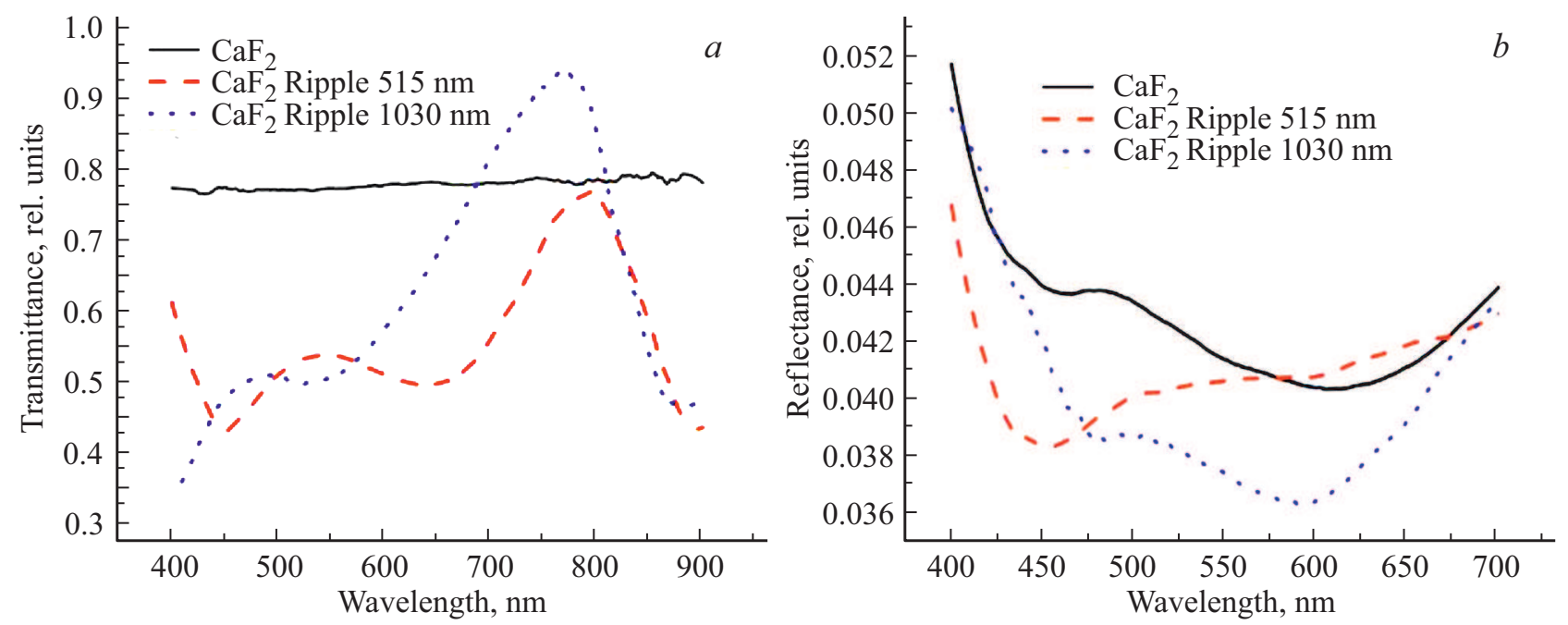

Рис. 5. Спектры пропускания $(a)$ и отражения $(b)$ участков модифицированных областей, записанных на поверхности фторида кальция при линейном направлении вектора поляризации. 
в результате чего был получен кристалл $\mathrm{CaF}_{2}: \mathrm{Ce}$ c неизвестной концентрацией Се.

С помощью диафрагмы размером $0.1 \mathrm{~mm}$ с разрешением $2.5 \mu \mathrm{m}$ была выполнена оптическая микроскопия отражения и пропускания на модифицированных участках по сравнению с эталонной необработанной поверхностью.

Значительно уменышился коэффициент пропускания модифицированного участка в синей области, это можно связать с рассеянием света из-за абляционной эрозии поверхности. В красной области спектрального диапазона наблюдается оптическое просветление (рис. 5,a). Напротив, в спектрах отражения (рис. $5, b$ ) для структур наблюдается уменьшение отражательной способности в видимом спектральном диапазоне. Исходя из спектральных данных можно сказать, что на структурах возникает изменение коэффициента пропускания и отражения, которые связаны с дифракцией и рассеянием света на получаемых решетках.

\section{Заключение}

В этой работе мы исследовали возможность создания самоорганизующихся структур, которые могут обладать оптическими эффектами, на поверхности фторида кальция при фемтосекудной лазерной обработке. Период получаемых нанометровых структур хорошо согласуется с расчетным для дисперсионных кривых. Исходя из экспериментальных данных и результатов численного моделирования можно сделать выводы о том, что механизм образования субволновых решеток действительно связан с интерференцией между поверхностными плазмонами. Оптические эффекты при пропускании можно связать с дифракцией света на решетке и рассеянием света из-за абляционной эрозии поверхности.

\section{Финансирование работы}

Исследование выполнено за счет гранта Российского научного фонда (проект № 20-71-10103).

\section{Конфликт интересов}

Авторы заявляют, что у них нет конфликта интересов.

\section{Список литературы}

[1] Sudrie L., Couairon A., Franco M., Lamourox B., Prade B., Tzortzakis S., Mysyrowicz A. // Phys. Rev. Lett. 2002. V. 89. N 18. P. 186601.

[2] Tien A.C., Backus S., Kapteyn H., Murnane M., Morou G. // Phys. Rev. Lett. 1999. V. 82. N 19. P. 3883.

[3] Gattass R., Mazur E. // Nature Photon. 2008. V. 2. P. 219 225. doi 10.1038/nphoton.2008.47

[4] Giridhar M. S., Seong K., Schülzgen A., Khulbe P., Peyghambarian N., Mansuripur M. /Appl. Opt. 2004. V. 43. N 23. P. 4584-4589.
[5] Shimotsuma Y., Kazansky P.G., Qiu J., Hirao K. // Phys. Rev. Lett. 2003. V. 91. N 24. P. 247405.

[6] Chen F., de Aldana J.R.V. // Las. Phot. Rev. 2014. V. 8. N 2. P. 251-275.

[7] Shimotsuma Y., Sakakura M., Kazansky P.G., Beresna M., Qiu J., Miura K., Hirao K. // Advan. Mat. 2010. V. 22. N 36. P. 4039-4043.

[8] Hong M.H., Luk'Yanchuk B., Huang S. M., Ong T.S., Van L.H., Chong T.C. // App. Phys. A. 2004. V. 79. N 4. P. 791-794.

[9] Drevinskas R., Kazansky P.G. // APL Phot. 2017. V. 2. N 6. P. 066104.

[10] Zhang J., Gecevičius M., Beresna M., Kazansky P.G. // Phys. Rev. Let. 2014. V. 112. N 3. P. 033901.

[11] Kudryashov S., Danilov P., Rupasov A., Khonina S., Nalimov A., Ionin A., Krasin G., Kovalev M. // Opt. Mat. Exp. 2020. V. 10. N 12. P. 3291-3305.

[12] Zhang J., Gecevičius M., Beresna M., Kazansky P.G. // CLEO: Science and Innovations. Opt. Soc. of America, 2013. P. CTh5D. 9.

[13] Chen T., Zhang G., Wang Y., Li X., Stoian R., Cheng G. // Micromach. 2020. V. 11. N 7. P. 671.

[14] Zhao X., Shin Y.C. // Appl. Phys. A. 2011. V. 104. N 2. P. $713-$ 719.

[15] Bashir S., Rafique M.S., Husinsky W. // Nuc. Inst. and Meth. in Phys. Res. Sec. B: Beam Inter. with Mat. and Atoms. 2012. V. 275. P. 1-6.

[16] Rupasov A.E., Danilov P.A., Kudryashov S.I. //J. Phys.: Conf. Ser. 2020. V. 1692. N 1. P. 012011.

[17] Rupasov A.E., Danilov P.A., Smaev M.P., Kovalev M.S., Zolot'ko A.S., Ionin A.A., Kudryashov S.I. // Opt. Spectrosc. 2020. V. 128. P. 928-931.

[18] Papadopoulos A., Skoulas E., Tsibidis G.D., Stratakis E. // Appl. Phys. A. 2018. V. 124. N 2. P. 1-12.

[19] Sipe J.E., Young J.F., Preston J.S., van Driel H.M. // Phys. Rev. B. 1983. V. 27. N 2. P. 1141.

[20] Kudryashov S.I., Nastulyavichus A.A., Saraeva I.N., Rudenko A.A., Zayarny D.A., Ionin A.A. // Appl. Surf. Sci. 2020. V. 519. P. 146204.

[21] Klimov V. // Nanoplasmonics, CRC press, 2014.

[22] Palik E.D. // Handbook of optical constants of solids, Academic press, 1998. V. 3.

[23] Groeneveld R.H.M., Sprik R., Lagendijk A. // Phys. Rev. B. 1995. V. 51. N 17. P. 11433.

[24] Kudryashov S.I., Levchenko A.O., Danilov P.A., Smirnov N.A., Rudenko A.A., Melnik N.N., Busleev N.I., Ionin A.A. // Appl. Phys. Lett. 2019. V. 115. N 7. P. 073102.

[25] Martsinovskǐ G.A., Shandybina G.D., Smirnov D.S., Zabotnov S.V., Timoshenko V.Y., Kashkarov P.K. // Opt. Spectrosc. 2008. V. 105. N 1. P. 67-72.

[26] Cheng Y., Wang Y., Teng, F., Dong H., Chen L., Mu J., Miao H. // Mat. Res. Exp. 2018. V. 5. N 3. P. 036206. 